Relations industrielles

Industrial Relations

\title{
Statistical Information Supplement
}

Labour Disputes in the Province of Quebec from April to

September, 1949

\section{Jean-H. Gagné}

Volume 5, numéro 9, juin 1950

URI : https://id.erudit.org/iderudit/1026330ar

DOI : https://doi.org/10.7202/1026330ar

Aller au sommaire du numéro

\section{Éditeur(s)}

Département des relations industrielles de l’Université Laval

\section{ISSN}

0034-379X (imprimé)

1703-8138 (numérique)

Découvrir la revue

Citer cet article

Gagné, J.-H. (1950). Statistical Information Supplement: Labour Disputes in the Province of Quebec from April to September, 1949. Relations industrielles /

Industrial Relations, 5(9), 92-96. https://doi.org/10.7202/1026330ar

Tous droits réservés @ Département des relations industrielles de l’Université Laval, 1950
Ce document est protégé par la loi sur le droit d'auteur. L'utilisation des services d'Érudit (y compris la reproduction) est assujettie à sa politique d'utilisation que vous pouvez consulter en ligne.

https://apropos.erudit.org/fr/usagers/politique-dutilisation/ 


\section{LES CONFLITS DU TRAVAIL DANS LA PROVINCE DE QUEBEC, D'AVRIL A SEPTEMBRE 1949}

\author{
Complété par Jean H. Gagné
}

Sous cette rubrique, l'on présentera dans le Bulletin, par tranche de six mois, une compilation des activités du Service de conciliation et d'arbitrage de la province, donnant une image aussi exacte que possible des conflits du travail survenus dans la province dans la dite période, des efforts faits par les parties et par l'Etat pour les régler dans le sens de la justice et de Péquité, des résultats obtenus par l'application de nos lois du travail. Plusieurs détails y seront ajoutés.

Les chiffres et les renseignements qui ont servi de base à cette compilation sont officiels et nous ont été gracieusement fournis par le Service d'information du ministère provincial du travail. La compilation, la présentation de l'information en tableaux, les notes explicatives nous sont personnelles. Dans ce numéro de juin du Bulletin, nous présentons notre information en tableaux différents, se complétant les uns les autres, pour la période d'avril à septembre 1949. Il est évident que ces chiffres ne peuvent être interprétés d'une manière absolue, mais sont quand même révélateurs de la tâche accomplie par le Ministère provincial du travail dans le règlement des conflits du travail par l'application des lois qui régissent actuellement les relations patronales ouvrières. Il est inutile de souligner ici l'évidente bonne volonté des parties.

Ces chiffres nous révèlent aussi que les rapports entre les représentants du capital et du travail sont plutôt pacifiques dans notre province et même que, plus souvent qu'autrement, les conflits sont réglés dans la paix et après entente entre les parties elles-mêmes. Dans tous les tableaux, les sigles S, I, C, T sont utilisés pour désigner services publics, industrie, commerce et total; les sigles A, B, C, D, E, F, G, H utilisés dans les tableaux, section de l'affiliation des associations ouvrières impliquées dans les conflits, veulent dire A: syndicat local non-affilié; B: syndicat national nonaffilié; $C$ : syndicat international non-affilié; $D$ : Congrès des métiers et du travail du Canada; E: Fédération américaine du travail; F: Congrès canadien du travail; G: Congrès des organisations industrielles; $\mathbf{H}$ : Confédération des travailleurs catholiques du Canada, sauf dans le tableau I, où ces sigles ont une signification différente.

TABLEAU 1: Cas exigeant inquete. Tableau comparatif des établissements où le Service de conciliation et d'arbitrage de la province de Québec a faít enquête et indiquant le nombre de conflits survenus, le nombre d'établissements et d'employés affectés, d’avril à septembre 1949, Dar catégorie d'établissements, selon le mois.

\section{LABOUR DISPUTES IN THE PROVINCE OF QUEBEC FROM APRIL TO SEPTEMBER, 1949}

\author{
Completed by Jean H. GaGné
}

Under this heading we present, by six month periods, a review of the activities of the provincial Conciliation and Arbitration Service giving as exact as possible a picture of the labour disputes that have occurred in the province in the said period, of the parties and efforts to regulate them in a just and equitable spirit, of the results obtained by the application of our labour legislation. This study will go into considerable detail. The figures and other information used as the basis of the study are official, graciously furnished by the Information Branch of the Department of Labour, in collaboration with the provincial Conciliation and Arbitration Service. The compilation, the graphic presentation, and the explanatory notes are our own. In this June number of the Bulletin, then, we present the relevant information for the period of April to September, 1949, broken down into a series of complementary tables.

Obviously these figures cannot be taken as absolute, but they nevertheless reveal the work accomplished by the Department of Labour in regulating labour disputes by applying the laws which currently govern managementlabour relations. It is unnecessary to emphasize the obvious good will of the parties concerned in these disputes. These figures also show that capital and labour representatives get along rather amicably in our province, and even that disputes, more often than not, are settled in a peaceful atmosphere by an understanding between the parties themselves.

In the tables the various economic factors involved are designated by the following symbols. For all tables: S: public services; $I$ : industry; $C$ : commerce; $T$ : total. For the tables dealing with the affiliation of workers' associations implicated in the disputes, A: local non-affiliated union; B: national non-affiliated union; C: international non-affiliated union; D: Trades and Labor Congress of Canada (TLCC); E: American Federation of Labour (AFL); F: Canadian Congress of Labour (CCL); G. Congress of Industrial Organizations ( $\mathrm{CIO}$ ); $\mathrm{H}$ : Canadian and Catholic Confederation of Labour, Inc. (CCCL), except in table I where these symbols have a different meaning.

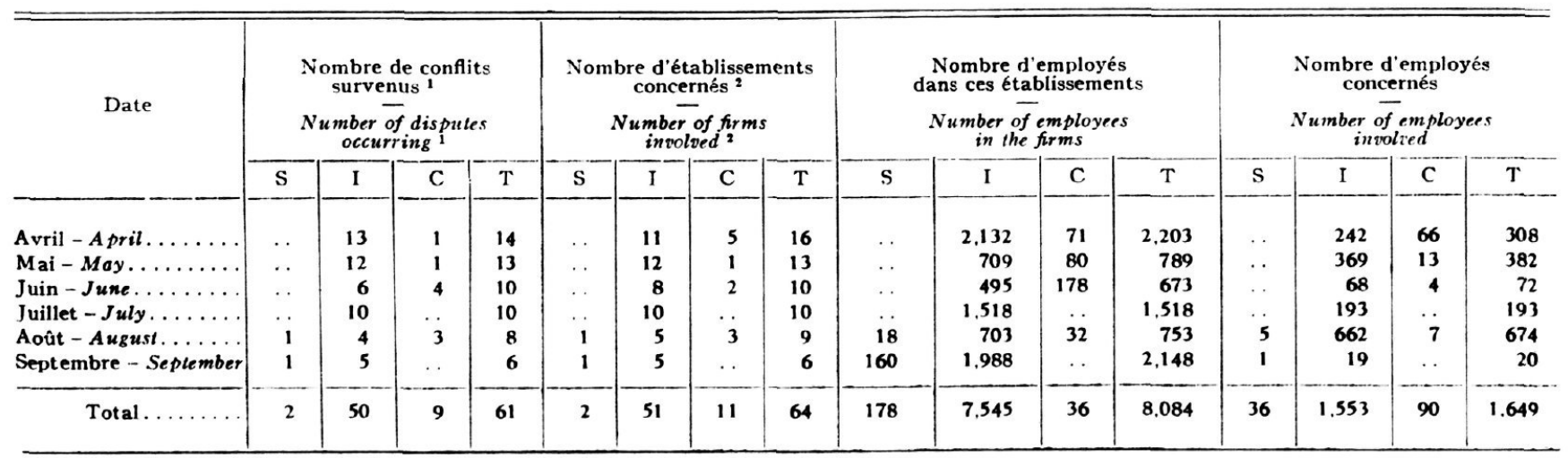

Notes: (1) A noter que le même établissement peut revenir plus doune Pois au cours du même mois, ou d'un mois à l'autre, parce qu'il y aura eu
plusieurs conflits différents entre les mêmes parties dans ces périodes. Le nombre total d'établissements différents est de 58.

(2) A noter qu'un même conflit peut affecter plusieurs établissements. C'est l'explication de la différence qui existe entre le total des conflits survenus et le total des établissements concernés au cours du même mois, ainst que pour le nombre total des conflits pendant les six mois étudíéc.
TABLE I: Cases requiring Investigation. Comparative table of firms in which the Conciliation and Arbitration Service of the Province of Quebec made an inrestigation, indicating the number of conflicts occurring. the number of firms and emplosees affected, according to type of firm, by months from April to September, 1949 
TABLEAU 1: Cas oxigeant enquéte (suite). Nombre total et fréquence des causes de conflits, et leur répartition par catégorie d'établissements, dans les cas où le Service de coneiliation et ü'arbitrage a fait enquête, selon le moís d.avril à septemabre 1949 .
TABLE I: Cases requiring investigation. Total number, frequency of causes, and distribution of disputes, according to type of firm, by months, from April to September, 1949 .

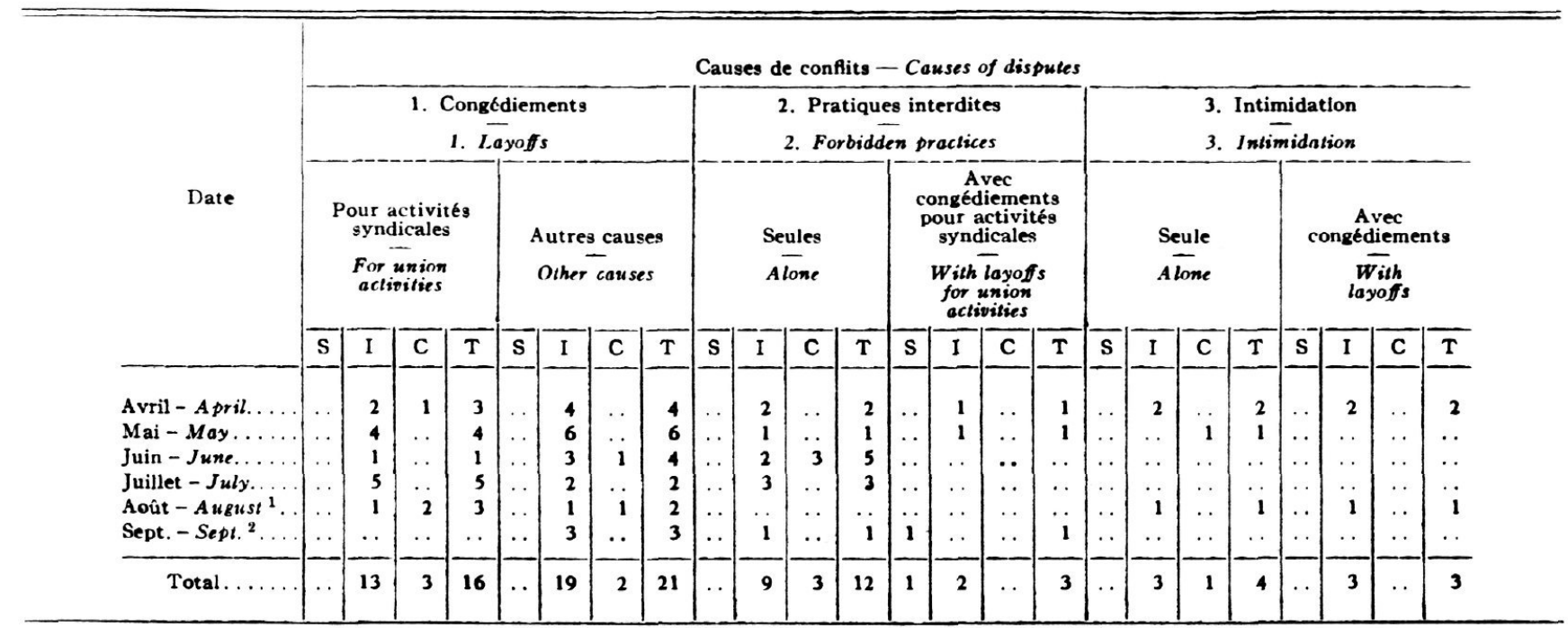

Notes: (1) Dans ce mois, un cas spécial à ajouter dans la eatégorie services publics, pour cause de "refus de réengager"; (2) Dans ce mois, un cas spéclal à ajouter dans la catégori. industrí, pour cause de "démission d'employés comme membres de l'union".

Dans les conflits du travail survenus dans la période étudiée, où l'intervention du Service de conciliation et d'arbitrage a été requise, la partie requérante est la partie ouvrière, sauf dans deux (2) cas, où c'est la partie patronale qui réclame l'aide du Service. Dans l'un, la cause du conflit provient du fait de la démission de 12 employés comme membres de l'union, dans un établissement industriel qui compte 13 employés. Dans l'autre, le conflit a pour cause, des pratiques interdites de la part d'une union en formation dans un établissement industriel de 80 employés.

TABLEAU 1: Cas exigeant enquâte (suite). Tableau indiquant le nombre de conflits survenus, les associations ourrières intéressées, le nombre de conflits réglés ou référés ì la Commission des relations ouvrières du Ministère provincial du travail, selon le mois, d'acril à septembre 1949.
N.B. (1) Special case this month in the sphere of public services, because of "refusal to rehire". (2) Special case this month in the sphere of in
dustry, because of "resignation of employees as members of the union".

\begin{tabular}{|c|c|c|c|c|c|c|c|c|c|c|c|c|c|c|c|}
\hline \multirow{3}{*}{ Date } & \multirow{2}{*}{\multicolumn{7}{|c|}{$\begin{array}{l}\text { Associations ouvrières intéressees } \\
\text { Workers' associations involved } \\
\begin{array}{c}\text { Affiliation ' } \\
\text { Afliation 1 }\end{array}\end{array}$}} & \multicolumn{8}{|c|}{$\begin{array}{l}\text { Résultats des enquetes } \\
\text { Résults of investigations }\end{array}$} \\
\hline & & & & & & & & \multicolumn{4}{|c|}{$\begin{array}{l}\text { Conflits réglés } \\
\text { Disputes settled }\end{array}$} & \multicolumn{4}{|c|}{$\begin{array}{l}\text { Ref. a } \mathrm{C}_{\mathrm{R} \mathrm{O}^{\prime}} \\
\text { Ref. } 10 \overline{\mathrm{L}} \mathrm{R} \mathrm{B}^{\prime}\end{array}$} \\
\hline & $\mathbf{A}$ & B & $\mathrm{C}$ & D & $\mathbf{E}$ & $\mathbf{F}$ & $\mathbf{T}$ & $\mathbf{s}$ & I & C & $T$ & $\mathbf{s}$ & $I$ & C & $\mathrm{T}$ \\
\hline 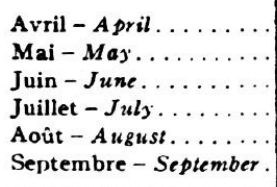 & $\begin{array}{l}\cdots \\
4 \\
2 \\
\cdots \\
\cdots\end{array}$ & $\begin{array}{l}\cdots \\
\cdots \\
\cdots \\
\because \\
1 \\
\cdots\end{array}$ & $\begin{array}{l}4 \\
1 \\
4 \\
2 \\
3 \\
1\end{array}$ & $\begin{array}{l}2 \\
2 \\
\cdots \\
\cdots \\
\cdots \\
\cdots\end{array}$ & $\begin{array}{l}1 \\
2 \\
1 \\
1 \\
1 \\
1\end{array}$ & $\begin{array}{l}7 \\
4 \\
1 \\
5 \\
3 \\
4\end{array}$ & $\begin{array}{r}14 \\
13 \\
10 \\
10 \\
8 \\
6\end{array}$ & $\begin{array}{l}\cdots \\
\cdots \\
\cdots \\
\cdots \\
\cdots \\
1\end{array}$ & $\begin{array}{l}8 \\
4 \\
2 \\
2 \\
2 \\
3\end{array}$ & $\begin{array}{l}\cdots \\
1 \\
2 \\
\cdots \\
1 \\
\cdots\end{array}$ & $\begin{array}{l}8 \\
5 \\
4 \\
2 \\
3 \\
4\end{array}$ & $\begin{array}{l}\cdots \\
\cdots \\
\cdots \\
\cdots \\
1 \\
\cdots\end{array}$ & $\begin{array}{l}5 \\
8 \\
4 \\
8 \\
3 \\
2\end{array}$ & $\begin{array}{c}1 \\
\cdots \\
2 \\
\ddot{1} \\
\ldots\end{array}$ & $\begin{array}{l}6 \\
8 \\
6 \\
8 \\
5 \\
2\end{array}$ \\
\hline Total.......... & 10 & 1 & 15 & 4 & 7 & 24 & 61 & 1 & 21 & 4 & 26 & 1 & 30 & 4 & 35 \\
\hline
\end{tabular}

Notes: (1) A, pour syndicat local non-affilié: B, pour Congrès des métiers et du travail du Canada (CMTC): C, nour Fédération américaine du travail (FAT): D, pour Congrès canadien du travail (CCT): E, pour Congrès des organisations industrielles (CIO): F. Confédération des travailleurs catholiques du Canada ((TCC).
In almost every labour dispute occurring during the period studied, where the intervention of the Conciliation and Arbitration Service was sought, the party requesting it was the workers, except in two cases in which management requested the aid of the Service. In one of these the cause of the confllict was the quitting of 12 employees as members of the union in an industrial establishment that counted only 13 employees. In the other, the conflict arose from forbidden practices on the part of a union organizing in an industrial enterprise of 80 employees.

TABLE 1: Cases requiring investigation. Number of disputes occurring workers' associations involved, number of disputes settled or referred to the Labour Relations Board of the Province of Quebec, by months, from April to September, 1949 . 
TABLEAU II: Cas portés à la conciliation. Nombre de conflits du travall qui furent portés à la conciliation, nombre d'établissements concernés, nombre d'employés dans ces derniers et nombre d'employés intéressés, par catévorie d'entreprises, selon le mois, d'avril à septembre 1949, dans la province de Québec. TABLE II: Cases submitted for coneiliation. Number of labour disputes
brought forward for concillation, number of firms involved, number of employees in theso firms, and number of employees involved, according to type of firm, by months, from April to September 1949, in the Province of Quebec.

\begin{tabular}{|c|c|c|c|c|c|c|c|c|c|c|c|c|c|c|c|c|}
\hline \multirow[t]{2}{*}{ Date } & \multicolumn{4}{|c|}{$\begin{array}{c}\text { Nombre de } \\
\text { conflits survenus } \\
\text { Number of } \\
\text { disputes occuring }\end{array}$} & \multicolumn{4}{|c|}{$\begin{array}{c}\text { Nombre d'établissements } \\
\text { concernés } 1 \\
\text { Number of } \\
\text { firms involved 1 }\end{array}$} & \multicolumn{4}{|c|}{$\begin{array}{c}\text { Nombre d'employés } \\
\text { dans ces établissements } \\
\text { Number of employees } \\
\text { in the firms }\end{array}$} & \multicolumn{4}{|c|}{$\begin{array}{c}\text { Nombre d'employés } \\
\text { concernés } \\
\text { Number of employees } \\
\text { involved }\end{array}$} \\
\hline & $\mathrm{s}$ & I & $\mathrm{C}$ & $T$ & s & I & $\mathrm{C}$ & $\mathrm{T}$ & $\mathrm{s}$ & I & $\mathrm{C}$ & $\mathrm{T}$ & $\mathrm{s}$ & I & $\mathrm{C}$ & $\mathrm{T}$ \\
\hline Avril -April . . . . . . . & 3 & 40 & 6 & 49 & 3 & 53 & 55 & 111 & 306 & 13.608 & 2,613 & 16,527 & 123 & 8,770 & 2,179 & 11,072 \\
\hline Août - A ugust ........ & .. & 17 & 4 & 21 & .. & 63 & 24 & 87 & & 10,353 & 764 & 11,127 & & 2,441 & 268 & 2,709 \\
\hline Septembre-September.. & 1 & 22 & 3 & 26 & 1 & 379 & 3 & 2,598 & 350 & 34,372 & 111 & 34,833 & 210 & 24,154 & 54 & 24,418 \\
\hline Total........... & 8 & 137 & 26 & 171 & 12 & 577 & 314 & 3,118 & 3,060 & 76,489 & 6,069 & 85,628 & 1,457 & 49,372 & 4.701 & 55,530 \\
\hline
\end{tabular}

Notes: (1) Pour fin dinterprétation, ì noter que, dans la section intitulée "No. d'établissements concernés", dans le nombre total 577, 246 6́tabllssements sont impliqués dans le même conflit et font partie de la même industrie, soit l'industrie de la chaussure de Québec.

TABLEAU II: Cas portés à la conciliation (suite). Associations ourrières intéressées, d'après l'affiliation, nature de l'intervention du Service de conciliation et d'arbitrage du Ministère du travail, et résultats de la conciliation gouvernementale, par catégorie d'établissements, selon le mois, d'avril a sepgouvernementale, par catégorie d'établissem
tembre 1949 , dans la province de Québec.
N.B. (1) For proper interpretation, it must be noted in the column dealing with the "No. of firms involved" the total for industries, 577, include 246 which were implicated in the same dispute and which are all part of the same industry, namely, the shoe industry of Quebec.

TABLE II: Cases submitted for conciliation. Workers' association involved (according to affiliation), type of intervention by the Conclliation and Arbitration Service of the Department of Labour, and results of government conciliation, according to type of firm, by months, from April to September, 1949, in the Province of Quebec.

\begin{tabular}{|c|c|c|c|c|c|c|c|c|c|c|c|c|c|c|c|c|c|c|c|c|c|c|c|c|c|c|c|c|c|c|}
\hline \multirow{3}{*}{ Date } & \multicolumn{9}{|c|}{$\begin{array}{l}\text { Associations ouvrières intéressées } \\
\text { Workers' associations involved }\end{array}$} & \multicolumn{9}{|c|}{$\begin{array}{c}\text { Nature de l'intervention } \\
\text { Type of intervention }\end{array}$} & \multicolumn{12}{|c|}{$\begin{array}{l}\text { Résultats de la conciliation } \\
\text { Results of conciliation }\end{array}$} \\
\hline & \multicolumn{9}{|c|}{$\begin{array}{l}\text { Affiliation }{ }^{1} \\
\text { Affliation }^{1}\end{array}$} & \multicolumn{3}{|c|}{$\begin{array}{l}\mathrm{LRO}^{2} \\
\bar{L}_{\mathrm{R} A^{2}}\end{array}$} & \multicolumn{6}{|c|}{$\begin{array}{l}\mathrm{L}^{\mathrm{DO}} \\
T \overline{D A}\end{array}$} & \multicolumn{4}{|c|}{$\begin{array}{l}\text { Conflits réglés } \\
\text { Disputes settled }\end{array}$} & \multicolumn{4}{|c|}{$\begin{array}{l}\text { Indécis } \\
\text { Disputes } \\
\text { undeatided }\end{array}$} & \multicolumn{4}{|c|}{$\begin{array}{l}\text { Arbitrage } \\
\text { Arbitration }\end{array}$} \\
\hline & A & B & C & D & E & F & G & $\boldsymbol{H}$ & $T$ & $N$ & $\boldsymbol{R}$ & $T$ & $N$ & $\boldsymbol{R}$ & G & $\mathrm{co}$ & PA & $T$ & $\mathbf{S}$ & I & C & $T$ & $\mathbf{S}$ & $I$ & C & $T$ & $s$ & I & C & $T$ \\
\hline $\begin{array}{l}\text { Avril-A pril } \\
\text { Mai - May. } \\
\text { Juin - June. } \\
\text { Juillet-July } \\
\text { Août - Aug. } \\
\text { Sept. - Sept. }\end{array}$ & $\begin{array}{l}1 \\
1 \\
3 \\
1 \\
1\end{array}$ & $\begin{array}{l}3 \\
1 \\
1 \\
1 \\
\ldots \\
\ldots\end{array}$ & $\begin{array}{l}3 \\
\cdots \\
\ddot{2} \\
\ldots \\
\ldots\end{array}$ & $\begin{array}{l}2 \\
2 \\
3 \\
\cdots \\
\ddot{2}\end{array}$ & $\begin{array}{l}7 \\
7 \\
4 \\
1 \\
6 \\
7\end{array}$ & $\begin{array}{l}1 \\
0 \\
1 \\
1 \\
2\end{array}$ & $\begin{array}{r}8 \\
2 \\
8 \\
1 \\
1 \\
\cdots\end{array}$ & $\begin{array}{r}24 \\
15 \\
11 \\
7 \\
13 \\
16\end{array}$ & $\begin{array}{l}49 \\
27 \\
34 \\
16 \\
22 \\
28\end{array}$ & $\begin{array}{r}13 \\
9 \\
7 \\
2 \\
4 \\
8\end{array}$ & $\begin{array}{r}21 \\
14 \\
18 \\
9 \\
13 \\
12\end{array}$ & $\begin{array}{l}34 \\
23 \\
25 \\
11 \\
17 \\
20\end{array}$ & $\begin{array}{c}\ddot{9} \\
1 \\
\cdots \\
\cdots \\
\ldots\end{array}$ & $\begin{array}{r}\mathbf{5} \\
2 \\
\ddot{3} \\
\cdots \\
\cdots\end{array}$ & $\begin{array}{l}9 \\
2 \\
5 \\
2 \\
4 \\
6\end{array}$ & $\begin{array}{l}\cdots \\
\cdots \\
\cdots \\
\cdots \\
\cdots \\
\cdots\end{array}$ & $\begin{array}{c}1 \\
1 \\
\cdots \\
1 \\
\ldots \\
\ldots\end{array}$ & $\begin{array}{r}15 \\
14 \\
6 \\
6 \\
4 \\
6\end{array}$ & \begin{tabular}{c|}
3 \\
2 \\
1 \\
1 \\
$\cdots$ \\
$\ldots$
\end{tabular} & $\begin{array}{r}25 \\
9 \\
14 \\
5 \\
8 \\
6\end{array}$ & $\begin{array}{r}2 \\
4 \\
3 \\
2 \\
2\end{array}$ & $\begin{array}{r}30 \\
15 \\
18 \\
8 \\
8 \\
8\end{array}$ & $\begin{array}{l}\ldots \\
\ldots \\
\ldots \\
\cdots \\
\ldots \\
\ldots\end{array}$ & $\begin{array}{r}6 \\
4 \\
1 \\
\cdots \\
\cdots \\
1\end{array}$ & $\begin{array}{c}1 \\
\ldots \\
\ldots \\
\ldots \\
\ldots \\
\ldots\end{array}$ & $\begin{array}{r}7 \\
4 \\
1 \\
\ldots \\
\cdots \\
1\end{array}$ & $\begin{array}{c}\ldots \\
\ldots \\
\ldots \\
\ldots \\
\ldots \\
\ldots\end{array}$ & $\begin{array}{r}10 \\
5 \\
9 \\
7 \\
6 \\
2\end{array}$ & $\begin{array}{c}\ldots \\
\cdots \\
1 \\
\cdots \\
\ldots \\
\ldots\end{array}$ & $\begin{array}{r}10 \\
5 \\
10 \\
7 \\
6 \\
2\end{array}$ \\
\hline Total. . & 7 & 6 & 5 & 9 & 32 & 11 & 20 & 86 & 176 & 43 & 87 & 130 & 10 & 10 & 28 & . & 3 & 51 & 7 & 67 & 13 & 87 & .. & 12 & 1 & 13 & $\cdots$ & 39 & I & 40 \\
\hline
\end{tabular}

Notes: (1) Parfois dans un même établissement, 11 y a deux unions intéressées. Le plus souvent ce sont les lettres $F$ et $F, F^{\prime}$ et $G$ quí se répètent. soit des unions affillées à la Fédération américaine du travail et au Congrès canadien du travail, ou à ce dernier et au Congrès des organisations industrielles.

(2) LRO signifie Loi des relations ouvrières de Québec et LDO signifie Loi des différends ouvriers de Québec.

(3) N, signifie nouvelle convention; $R$, renouvellement; G, griefs ; Co, clause ouverte: PA, conclliation post-arbitrale.

(4) Les conflits commencés dans un mols donné ont pu être réglés dans un autre mois. Dans les chlffres ci-haut indiqués, les conflits sont additionnés dans chaque mois comme s'ils se fussent réglés dans ce même mols. Mais plusieurs conflits qui ont commencé dans chacun de ces mois, sont encore au stage de la conciliation au mois de septembre, fin de is période étudiée. En avril, 2 sont encore au stage de la conciliation en septembre; en mai, 3; en juin, 2; en julllet, 2 ; en aout, 7 ; et en septembre, 19. La presque totalité de ces conflits encore en vole de règlement en septembre sont dans les établissements industriels.

Il est intéressant de noter que la plupart des conflits du travail sont réglés au stage de la conciliation, ce qui est tout à l'honneur des représentants des parties en présence et de ceux du Service de conciliation et d'arbitrage de la province de Québec, sections de Montréal et de Québec.

Il serait intéressant aussi de donner en détail quels ont été les sujets en litige dans ces conflits, mais ce serait trop long. Disons simplement que les principaux d'entre
N.B. (1) Sometimes there are two unions involved in the same firm. This happens most often in the firms in columns $E, F$ and $G$.

(2) LRA, Labour Relations Act (Quebec).

(3) TDA, Quebec Trade Disputes Act; N, new agreement; $R$, renewal G, grievances; CO, open clause; PA, post-arbitration conciliation.

(4) Disputes begun in a given month can have been settled in another month. In the above figures the disputes are added up. in each month as if they had been settled in the same month. Several disputes from each month are still in the stage of conciliation at the month of September, end month are stin in the stage of conciliation at the month of September, end the period studied. Thus, of disputes beginning in April, 2 are stil] at the conciliation stage by September; of those in May, 3; of those in June, 2; of those in July, 2; of those in August, 7; and of those in Sep September were in industrial establishments.

It is worthy of note that the greater part of the labour disputes were settled at the conciliation stage, a tribute to the representatives of the parties present and to the officials of the Montreal and Quebec sections of the Conciliation Service of the Province of Quebec, who lent their professional assistance to bring about an entente between the parties concerned.

It would be interesting, also, to list in detail the subjects at issue in the disputes, but space does not per- 
eux, dans tous les conflits soumis au Service, ont des origines très variées.

Voici la répartition des causes de conflit, dans 171 cas: 83 (environ $48.5 \%$ ), demandes d'augmentations de salaires; 66 (environ $38.6 \%$ ), revision de toute la convention existante, plus nouvelles demandes; 33 (environ 19.3\%), vacances payées; $32(18.7 \%)$, durée du travail; 29 (environ 16.4\%), sécurité syndicale; 27 (environ 15.8\%), congés payés; 16 (environ $9.3 \%$ ), classification des tàches; 14 (environ $8.2 \%$ ), promotions, permutations, licenciements et congédiements; 12 (environ $7.0 \%$ ), surtemps; 8 , ancienneté; 8 (environ $4.6 \%$ ), jours fériés; 6 (environ $3.5 \%$ ), sécurité sociale (à l'exception des plans de pension). Dans un cas, l'objet du litige était la contestation des droits de la direction par les représentants ouvriers. Ce sont là les principaux sujets de conflits avec leur fréquence.

TABLEAU III: Cas portés à l'arbitrage. Nombre de conflits, nombre d'établissements concernés, nombre d'employés affectés, d'arril à septembre 1949, dans la province de Québec. mit. We must be content merely to note that the principal points of disagreement in all the disputes submitted to the Service are distributed as follows:

Of 171 disputes: 83 , about $48.5 \%$, were about wage increases; 66 , about $38.6 \%$, involved the whole of the existing Agreement plus new demands; 33 , about $19.3 \%$, were about paid vacations; 32 , about $18.7 \%$, about working hours; 29 , about $16.4 \%$, about union security; 27 , about $15.8 \%$, about paid leave of absence; 16 , about $9.3 \%$, in connection with job classification; 14 , about $8.2 \%$, in connection with promotions, transfers, dismissals and layoffs; 12 , about $7.0 \%$, about demands for overtime; 8 about seniority and 8 about holidays, about $4.6 \%$; 6 , about $3.5 \%$, in connection with social security (excepting pension plans). One case involved a dispute about jurisdictional rights. These are the principal subjects at issue, and their frequency.

TABLE III: Cases submitted for arbitration. Number of disputes, number of firms involved, and number of employees affected, from April to september. 1949, in the Province of Quebec.

\begin{tabular}{|c|c|c|c|c|c|c|c|c|c|c|c|c|c|c|c|c|}
\hline \multirow[t]{2}{*}{ Date } & \multicolumn{4}{|c|}{$\begin{array}{l}\text { Nombre } \\
\text { de conflits } \\
\text { Number } \\
\text { of disputes }\end{array}$} & \multicolumn{4}{|c|}{$\begin{array}{c}\text { Nombre d'etablissements } \\
\text { concernes } \\
\text { Number of } \\
\text { firms involved }\end{array}$} & \multicolumn{4}{|c|}{$\begin{array}{l}\text { Nombre d'employes } \\
\text { dans les établissements } \\
\text { Number of employees ... } \\
\text { in ihe firms }\end{array}$} & \multicolumn{4}{|c|}{$\begin{array}{l}\text { Nombre d'employés } \\
\text { concernés } \\
\begin{array}{c}\text { Number of employees } \\
\text { involved }\end{array}\end{array}$} \\
\hline & $\mathrm{s}$ & I & C & $\mathrm{T}$ & $\mathrm{s}$ & I & C & $T$ & $\mathbf{s}$ & I & C & $\mathrm{T}$ & $\mathbf{s}$ & I & C & $\mathrm{T}$ \\
\hline 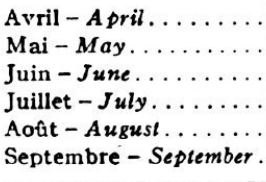 & $\begin{array}{l}11 \\
\cdots \\
\cdots \\
\cdots \\
\cdots \\
\cdots\end{array}$ & $\begin{array}{r}26 \\
3 \\
6 \\
10 \\
7 \\
7\end{array}$ & $\begin{array}{l}2 \\
\cdots \\
1 \\
\cdots \\
\cdots \\
\cdots\end{array}$ & $\begin{array}{r}39 \\
3 \\
7 \\
10 \\
7 \\
7\end{array}$ & $\begin{array}{l}12 \\
\cdots \\
\cdots \\
\cdots \\
\cdots \\
\cdots\end{array}$ & $\begin{array}{r}445 \\
3 \\
6 \\
15 \\
17 \\
126\end{array}$ & $\begin{array}{l}54 \\
\cdots \\
4 \\
\cdots \\
\cdots \\
\cdots\end{array}$ & $\begin{array}{r}511 \\
3 \\
10 \\
15 \\
17 \\
26\end{array}$ & $\begin{array}{r}17,072 \\
\ldots \\
\ldots \\
\ldots \\
\ldots \\
\ldots\end{array}$ & \begin{tabular}{r|}
22,206 \\
1,541 \\
5,804 \\
3,488 \\
2,408 \\
2,658
\end{tabular} & $\begin{array}{r}1.240 \\
\ldots \\
53 \\
\ldots \\
\ldots \\
\ldots\end{array}$ & $\begin{array}{r}40,518 \\
1,541 \\
5.857 \\
3,488 \\
2,408 \\
2,658\end{array}$ & $\begin{array}{r}3,641 \\
\ldots \\
\ldots \\
\ldots \\
\ldots \\
\ldots\end{array}$ & $\begin{array}{r}17,945 \\
1,279 \\
4,490 \\
3,169 \\
1,711 \\
2,326\end{array}$ & $\begin{array}{l}\ddot{5} \\
\cdots \\
\cdots \\
\cdots\end{array}$ & $\begin{array}{r}22,718 \\
1,279 \\
4,543 \\
3,169 \\
1,711 \\
2,326\end{array}$ \\
\hline Total. & 11 & 59 & 3 & 73 & 12 & 612 & 58 & 682 & 17,072 & 38,105 & 1,293 & 56.470 & 3,641 & 30,920 & 1,185 & 35.746 \\
\hline
\end{tabular}

TABLEAU III: Cas portés à l'arbitrage (suite). Associations ourrières intéresressées par l'affiliation; nombre de tribunaux formés, choix du président, ressées par l'affiliation; nombre de tribunaux formés, choix du président,
nombre de séances, nature des sentences rendues, nombre de sentence nonnombre de séances, nature des sentences rendues, nombre de sent
rendues, d'arril à septembre 1949, dans la province de Québec.
TABLE III: Cases submitted for arbitration. Workers' Associations involved by affiliation; number of tribunals formed, choice of president, number of meetings, type of decisions rendered, number of decisions not rendered from April to September, 1949, in the Province of Quebec.

\begin{tabular}{|c|c|c|c|c|c|c|c|c|c|c|c|c|c|c|c|c|c|c|}
\hline \multicolumn{11}{|c|}{$\begin{array}{l}\text { Associations ouvrières intéressées } \\
\text { Workers' associations involved }\end{array}$} & \multirow{4}{*}{$\begin{array}{c}\begin{array}{c}\text { Nombre } \\
\text { de } \\
\text { seances } \\
\text { Number } \\
\text { of } \\
\text { meetings }\end{array} \\
\text { (a) }\end{array}$} & \multicolumn{7}{|c|}{$\begin{array}{l}\text { Sentences arbitrales ' } \\
\text { Arbitration decisions ' }\end{array}$} \\
\hline \multirow{2}{*}{\multicolumn{9}{|c|}{$\begin{array}{l}\text { Affiliation } \\
\text { Affitiation }\end{array}$}} & \multirow{2}{*}{\multicolumn{2}{|c|}{$\begin{array}{l}\text { Président } \\
\text { désigné } \\
\text { President } \\
\text { designated }\end{array}$}} & & \multicolumn{6}{|c|}{ rendues-rendered } & \multirow{3}{*}{$\begin{array}{c}\text { Atten- } \\
\text { dues } \\
\text { en } \\
\text { sep- } \\
\text { tembre } \\
\text { Still } \\
\text { pendin } \\
\text { in } \\
\text { Sep- } \\
\text { lember }\end{array}$} \\
\hline & & & & & & & & & & & & \multicolumn{2}{|c|}{$\begin{array}{l}\text { exécutoire } \\
\text { by executive }\end{array}$} & \multirow{2}{*}{$\begin{array}{c}\text { una- } \\
\text { nime } \\
\overline{u n-} \\
\text { ani- } \\
\text { mous- } \\
l y\end{array}$} & \multicolumn{2}{|c|}{$\begin{array}{l}\text { majoritaire } \\
\text { by majority 4 }\end{array}$} & \multirow{2}{*}{$\mathrm{T}$} & \\
\hline$A$ & B & $\mathrm{C}$ & $\mathrm{D}$ & $\mathbf{E}$ & $\mathrm{F}$ & G & $\mathbf{H}$ & $\mathbf{T}$ & Arb. & Min. & & $\begin{array}{l}\text { cont. } \\
\text { cont. } \\
\text { eng. }\end{array}$ & $\begin{array}{l}\text { la loi } \\
\text { acc. } \\
\text { to the } \\
\text { act }\end{array}$ & & $\frac{\text { pat. }}{\text { man. }}$ & $\frac{\text { ouv. }}{\text { lab. }}$ & & \\
\hline & 3 & $\ldots$ & 4 & 10 & 1 & 5 & 17 & 39 & 22 & 17 & 714 & $\ldots$ & 4 & 16 & 4 & 13 & 33 & 5 \\
\hline 1 & .. & . & 1 & $\ldots$ & .. & $\ldots$ & 1 & 3 & 2 & 1 & 16 & .. & $\ldots$ & $\ldots$ & 1 & .. & 1 & 2 \\
\hline 1 & $\ldots$ & $\ldots$ & 1 & $\ldots$ & . & 2 & 3 & 7 & 5 & 2 & 18 & 1 & $\ldots$ & 2 & $\ldots$ &.. & 2 & 5 \\
\hline 1 & .. & .. & .. & 1 & 2 & 4 & 2 & 10 & 10 & $\ldots$ & 18 & .. & $\ldots$ & 3 & .. & .. & 3 & 7 \\
\hline$\ldots$ &.. & 1 & .. & 1 & 1 & 1 & 3 & 7 & 6 & 1 & 4 & .. & $\ldots$ & $\ldots$ & .. & 1 & 1 & 6 \\
\hline .. & 1 & . & 2 & 3 & 1 & 1 & 1 & 6 & 5 & 1 & . & .. & .. & .. & .. & .. & .. & 7 \\
\hline 3 & 4 & 1 & 8 & 15 & 5 & 13 & 27 & 72 & 50 & 22 & 770 & 1 & 4 & 21 & 5 & 14 & 40 & 32 \\
\hline
\end{tabular}

Notes: (1) Arb, veut dire "par les arbitres"; Min., par le Ministre du travail.

(2) Dans l'interprétation de la différence entre le nombre total des sentences arbitrales et le nombre total des conflits, l'on doit tenir compte gue dans un conflit survenu en septembre, le tribunal n'était pas encore formé.

(3) Eng. cont. signifie, engagement contractuel. ouvrier.

(4) Arb. pat. signifie arbitre patronal; arb. ouv. signifie arbitre

Il serait intéressant de noter quels sont les principaux points des mandats confiés aux arbitres lors de la formation des tribunaux d'arbitrage. Mais comme l'énumération serait trop longue, nous n'en indiquerons que
N.B. (1) In interpreting the difference between the total number of arbi tration decisions and the total number of disputes, one must take account of the fact that for a dispute beginning in September, the tribunal was not yet set up.

(2) Arb, means "by the arbitrators"; Min, "by the Minister of
Labour". (3) Cont. eng. means "contractual engagement".

(4) Public Services Employees' Disputes Act.

(5) Man. means "employer's representative"; lab. means "employee's representative".

It would be interesting to list the principal points of the commissions entrusted to the arbitrators at the time of the setting up of the arbitration tribunals. But as the enumeration would be too long, we simply indicate a few. 
quelques-uns. Dans 50 cas sur 72, soit environ 69.5\%, il est question d'augmentations de salaires; dans 27 cas, soit environ $37.5 \%$, toute la convention existante et de nouvelles demandes sont en cause; dans 21 cas, soit environ $29.2 \%$, il est question de sécurité syndicale; dans 20 cas, soit environ $27.7 \%$, il est question de vacances payées; dans 16 cas, soit environ $22.2 \%$, il est question de la durée du travail; dans 12 cas, soit environ $18.1 \%$, il est question de surtemps; dans 11 cas, soit environ $15.3 \%$, il est question de congés payés; dans 10 cas, soit environ $13.9 \%$, de sécurité sociale, (à l'exception des plans de pension); dans 9 cas, soit environ $12.5 \%$, de classification des tâches; dans 7 cas, soit environ $9.7 \%$, il est question de procédures de règlements de griefs; le même pourcen. tage est dévolu aux points suivants: 1 cas pour ancienneté, durée et renouvellement de convention; dans seulemen ${ }^{+}$ 4 cas, soit $5.5 \%$ il est question de permutations, promo tions, congédiements et licenciements.
In 50 cases out of 72 , about $69.5 \%$, the question was one of wage increases; in 27 cases, about $37.5 \%$, the whole existing Agreement plus new demands; in 21 cases, about $29.2 \%$, union security; in 20 cases, about $27.7 \%$, paid vacations; in 16 cases, about $22.2 \%$, length of working hours; in 12 cases, about $18.1 \%$, overtime; in 11 cases, about $15.3 \%$, paid leave of absence; in 10 cases, about $13.9 \%$, social security (with the exception of pension plans); in 9 cases, about $12.5 \%$, job classification; in 7 cases, about $9.7 \%$, grievance procedures. The same percentage was found for cases involving seniority, and duration and renewal of the Agreement. Only 4 cases, about $5.5 \%$, dealt with transfers, promotions, layoffs and dismissals.
TABLEAU IV: Gròves dans la prevince de Qucbec, d'avril a septembre 1949: Nombre et nature des établissements concernés, nombre d'employés affectés. Nombre et nature des établissements concernés.
applliation des assoeiations ourrières Intéressées.
TABLE IV: Strikes in the Province of Quobee, from April to September, 1949. Number and type of firms involved, number of employees affeeted. workers. Number and type of

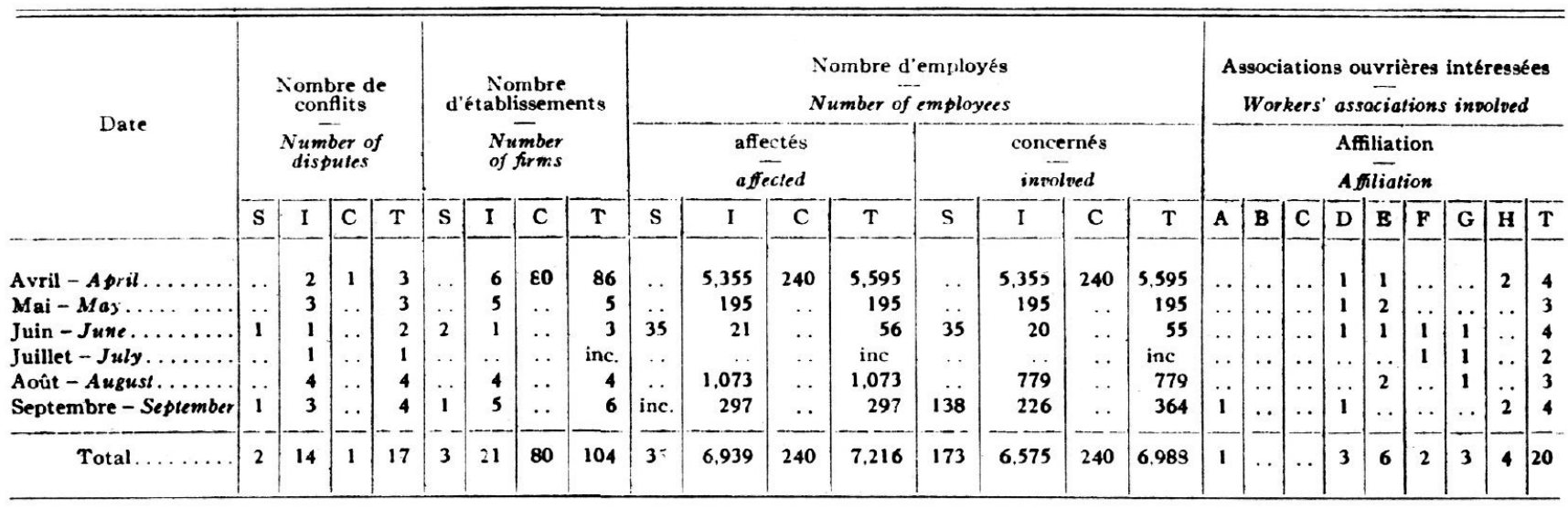

TABLEAU IV: Gròves dans la provinee de Québee, d'avril à septembre 1949 (suite). Nature de l'intersention, grèves légales ou illégales, moment du déclenchement de la grève, gain réalisé par les parties, pertes estimées en journées individuelles de travail
TABLE IV: Strikes in the Province of Quebec. From April to September. 1949. Type of intervention, legal or illegal strikes, time of beginning of strike, gains realized hy the parties, losses estimated in individual work days.

\begin{tabular}{|c|c|c|c|c|c|c|c|c|c|c|c|c|c|}
\hline \multirow{4}{*}{ Date } & \multirow{3}{*}{\multicolumn{2}{|c|}{$\begin{array}{c}\text { Intervention } \\
\text { gouverne- } \\
\text { mentale } \\
\text { Govern- } \\
\text { mental } \\
\text { interiention }\end{array}$}} & \multicolumn{6}{|c|}{ Procédures légales - Legal procedures } & \multicolumn{4}{|c|}{ Résultats - Results } & \multirow{4}{*}{$\begin{array}{c}\text { Pertes en } \\
\text { journés } \\
\text { individuelles } \\
\text { de travail }\end{array}$} \\
\hline & & & \multirow{3}{*}{$\begin{array}{c}\text { Sui- } \\
\text { vies } \\
\text { Fol- } \\
\text { lowed }\end{array}$} & \multicolumn{5}{|c|}{ Non suivies - Not followed } & \multirow{2}{*}{\multicolumn{2}{|c|}{$\frac{\text { Gain }}{\text { Gains }}$}} & \multirow{3}{*}{$\begin{array}{c}\text { Com- } \\
\text { promis } \\
\text { Com- } \\
\text { promise }\end{array}$} & \multirow{3}{*}{$\begin{array}{l}\text { Indé- } \\
\text { fini } \\
\text { Inde- } \\
\text { finite }\end{array}$} & \\
\hline & & & & \multicolumn{2}{|c|}{$\begin{array}{c}\text { Par qui }{ }^{2} \\
\text { Py whom 2 }\end{array}$} & \multicolumn{3}{|c|}{$\begin{array}{c}\text { Phase des } \\
\text { procédures } \\
\text { Phase of } \\
\text { procedures }\end{array}$} & & & & & \\
\hline & $\mathbf{F}$ & $\mathrm{S}^{1}$ & & $\frac{P}{L}$ & $\mathrm{P}_{\overline{\mathrm{M}}}^{\mathrm{P}}$ & $\mathbf{P}$ & $\mathrm{N}$ & $\mathrm{C}$ & $\frac{\mathbf{P}}{\mathbf{M}}$ & $\frac{\mathrm{O}}{\mathrm{L}}$ & & & \\
\hline Avril - April ${ }^{s} \ldots \ldots \ldots \ldots \ldots \ldots$ & . & 3 & 2 & 1 & . & $\ldots$ & . & 1 & . & 1 & $\ldots$ & $\mathbf{1}$ & 5,012 \\
\hline $\mathbf{M a i}-\mathbf{M a y} \ldots \ldots \ldots \ldots \ldots \ldots$ & . & 3 & . & 3 & . & 3 & . & $\ldots$ & . & .. & 2 & 1 & 205 \\
\hline Juin - June. . . . . . . . . . & . & 2 & . & 2 & . & . & 2 & . & . & $\ldots$ & 1 & 1 & 75 \\
\hline Juillet $-J_{u l y}{ }^{6} \ldots \ldots \ldots \ldots \ldots$ & . & 1 & 1 & . & . & . & . & inc. & & ve e & septembr & & .. \\
\hline Août - A ugust.$\ldots \ldots \ldots \ldots \ldots$ & . & 4 & . & . & 4 & 1 & 3 & . & $\ldots$ & $\ldots$ & 4 & $\ldots$ & 866 \\
\hline Septembre - Sepiember ${ }^{7} \ldots \ldots \ldots$ & . & 4 & 2 & . & 2 & . & 2 & . & . & 1 & .. & 2 & $2,513^{4}$ \\
\hline Total ............ & . & 17 & 5 & 6 & 6 & 4 & 7 & 1 & . & 2 & 7 & 5 & 8,671 \\
\hline
\end{tabular}

Notes: (1) Les sigles F. M. S, veulent dire: F. Ministère fédéral du trarail: S, le Service de conclliation et d'arbitrage du Ministère provincial du travail: (2) Les sigles PO et PP veulent dire respectivement par la partie ou vrière et par la partie patronale. (3) Phase des procédures où le conflít a śclaté. Les lettres $P, N$ et $\mathbf{C}$, signifient pourparlers, négociations et conciliation: (4) Les lettres $P$ et 0 signifient patronal et ourrier; (5) Dan ces chiffres, ceux de la grève de l'amiante n'y sont pas compris, parce qu'ils n'avaient pas encore été compilés a Ministère en septembre: (6) La grère commencée en ce mois se continue en septemlire: (7) Un des quatre cas de grève survenus en ce mois, n'est pas eneore réglé en septembre.
N. B. (1) The symbols $\mathrm{F}$ and $\mathrm{S}$ signify Federal Minister of Labour and Arbitration Service of the Department of Labour.

(2) The symbols $L$ and II mean "on the part of labour" and "on the part of management". (3) The letters $P, X$ and $C$ stand for discussion, negociations and conciliation. (4) The letter M stands for "manasement": the letter L for "labour". (5) The figures concerning the asbestos strike are not included in this table because the final result of that was still pending in September. (6) The strike occurring in this month continued in September. (7) One of the 4 cases of strikes in this month settled in September. 